OPEN ACCESS

Edited by:

Silvia R. Rogatto,

University of Southern Denmark,

Denmark

Reviewed by:

Jun Hatazawa,

Osaka University, Japan

Søren Rafaelsen,

University Hospital of Southern

Denmark, Denmark

*Correspondence:

Yong Beom Cho

yongbeom.cho@samsung.com

${ }^{t}$ These authors have contributed equally to this work and share first authorship

Specialty section:

This article was submitted to Gastrointestinal Cancers: Colorectal Cancer. a section of the journa Frontiers in Oncology

Received: 16 July 2021 Accepted: 14 September 2021 Published: 05 October 2021

Citation:

Pyo $D H$, Choi JY, Lee WY, Yun SH, Kim HC, Huh JW, Park YA, Shin JK and Cho YB (2021) A Nomogram for

Predicting Pathological Complete

Response to Neoadjuvant

Chemoradiotherapy Using

Semiquantitative Parameters Derived

From Sequential PET/CT in Locally

Advanced Rectal Cancer.

Front. Oncol. 11:742728.

doi: 10.3389/fonc.2021.742728

\section{A Nomogram for Predicting Pathological Complete Response to Neoadjuvant Chemoradiotherapy Using Semiquantitative Parameters Derived From Sequential PET/CT in Locally Advanced Rectal Cancer}

\author{
Dae Hee Pyo ${ }^{1+}$, Joon Young $\mathrm{Choi}^{2 \dagger}$, Woo Yong Lee ${ }^{1,3}$, Seong Hyeon Yun ${ }^{1}$, \\ Hee Cheol Kim ${ }^{1}$, Jung Wook Huh ${ }^{1}$, Yoon Ah Park ${ }^{1}$, Jung Kyong Shin ${ }^{1}$ \\ and Yong Beom Cho ${ }^{1,3,4 *}$
}

\begin{abstract}
1 Department of Surgery, Samsung Medical Center, Sungkyunkwan University School of Medicine, Seoul, South Korea, 2 Department of Nuclear Medicine, Samsung Medical Center, Sungkyunkwan University School of Medicine, Seoul, South Korea, ${ }^{3}$ Department of Health Sciences and Technology, Samsung Advanced Institute for Health Sciences \& Technology (SAIHST), Sungkyunkwan University, Seoul, South Korea, ${ }^{4}$ Department of Biopharmaceutical Convergence, Sungkyunkwan University, Seoul, South Korea
\end{abstract}

We evaluated the predictive value of semiquantitative volumetric parameters derived from sequential PET/CT and developed a nomogram to predict pathological complete response $(\mathrm{pCR})$ in patients with rectal cancer treated by neoadjuvant chemoradiotherapy ( $\mathrm{nCRT}$ ). From April 2008 to December 2013, among the patients who underwent nCRT, those who were taken sequential PET/CT before and after nCRT were included. MRI-based staging and semiquantitative parameters of PET/CT including standardized uptake value (SUV), metabolic tumor volume (MTV), and total lesion glycolysis (TLG) were evaluated before and after nCRT. Multivariable analysis was performed to select significant predictors to construct a nomogram. Sensitivity, specificity, accuracy, and area under the receiver operating characteristics curve (AUC) of the model were evaluated to determine its performance. Among 137 eligible patients, 17 (12.4\%) had pCR. All post-PET/CT parameters showed significant differences between pCR and non-pCR groups. Patients were randomly assigned to a training group (91 patients) and a validation group (46 patients). In multivariable analysis with the training group, post-CEA, post-MRI T staging, post-SUV $V_{\max }$, and post-MTV were significantly associated with $\mathrm{pCR}$. There was no significant pre-nCRT variable for predicting $\mathrm{pCR}$. Using significant predictors, a nomogram was developed. Sensitivity, specificity, accuracy, and AUC of the nomogram were $0.882,0.808,0.848$, and 0.884 with the training group and $0.857,0.781$, 0.783 , and 0.828 with the validation group, respectively. This model showed the better performance than other predictive models that did not contain PET/CT parameters. A nomogram containing semiquantitative post-PET/CT could effectively select candidates for organ-sparing strategies.

Keywords: locally advanced rectal cancer (LARC), neoadjuvant chemoradiotherapy, pathological complete response (pCR), PET/CT, nomogram 


\section{INTRODUCTION}

Colorectal cancer is one of the most commonly diagnosed malignancies. It is the major cause of cancer-related deaths in the world according to reports of the World Health Organization (1). In 2020, 732,210 new cases of rectal cancer were diagnosed with 339,022 deaths due to rectal cancer. The current standard treatment for non-metastatic locally advanced rectal cancer (LARC) is a preoperative or neoadjuvant long-course concurrent chemoradiotherapy (nCRT) followed by radical surgery at intervals of 8-12 weeks (2). After completion of nCRT, approximately $15-20 \%$ patients achieve pathological complete response (pCR) defined as an absence of any residual cancer cells (ypTxN0M0) in the surgical specimen $(3,4)$. Because radical surgery for rectal cancer causes significant morbidity and deteriorates patients' quality of life, causing fecal, urinary, or sexual dysfunction and permanent stoma in some cases, organsparing strategies such as "wait-and-see" (5-7) and transanal local excision (8-10), have been recently proposed. One of the most important prerequisites to select appropriate candidates for these conservative strategies is the construction of a reliable prediction model for $\mathrm{pCR}$ without pathological information of surgical specimens. Although many efforts have been made to identify robust clinical predictors for $\mathrm{pCR}$, any single modality has not been validated to present a sufficient predictive power. Although serum level of carcinoembryonic antigen (CEA) could be easily and rapidly evaluated, its false-positive rates cause concerns (11). Magnetic resonance imaging (MRI) has advantages on excellent spatial resolution enabling anatomical diagnosis for depth of tumor invasion and identification of lymph nodes (12). However, without diffusion and intravenous contrast MRI has a limited role in evaluating the viability of tumor.

$\mathrm{PET} / \mathrm{CT}$ is a well-established imaging modality for cancer evaluation. It is advantageous in presenting the physiological process of a tumor, thereby distinguishing the remained viable tumor tissue from the fibrosis induced by radiation. Recent studies have revealed that several semiquantitative metabolic and volumetric parameters derived from PET/CT, including metabolic tumor volume (MTV), total lesion glycolysis (TLG), and standardized uptake value (SUV), are significantly associated with therapeutic responses in several types of cancer (13-16).

In this study, we evaluated the predictive efficacy of semiquantitative metabolic and volumetric parameters derived from sequential PET/CT taken before and after nCRT in patients with LARC. In addition, we developed and validated a pCRpredicting nomogram incorporating $\mathrm{PET} / \mathrm{CT}$ parameters with other clinical features including CEA and MRI findings.

\section{MATERIALS AND METHODS}

\section{Patient Selection}

Among non-metastatic primary rectal cancer patients with clinical T3/T4 stage, or lymph node involvement treated with
nCRT followed by curative resection at Samsung Medical Center from April 2008 to December 2013, those who underwent sequential PET/CTs taken before and after nCRT were included in this study. All patients were staged with standard examinations at the initial workup, including digital rectal examination, endoscopic ultrasound, rigid proctoscopy, abdominopelvic computed tomography (CT), pelvic MRI, serum level of CEA, and PET/CT. After completion of nCRT, blood test for CEA, MRI, and PET/CT were performed. Informed consent was obtained from all participants to preserve their clinical data in a form of a database to use in future research regarding colorectal cancer. Data were extracted from the Clinical Data Warehouse Darwin-C of Samsung Medical Center for this study. This retrospective study design was approved by the Institutional Review Board (IRB) of Samsung Medical Center (Number: 2019-12-056).

\section{Neoadjuvant Chemoradiotherapy and Surgery}

The use of nCRT was decided by a multidisciplinary team consisting of colorectal surgeons, medical oncologists, and radiation oncologists. Radiation was administered to the whole pelvic field at a total dose of $50.4 \mathrm{~Gy}$ in 25 fractions. Chemotherapy was administered concurrently with radiation based on 5-fluorouracil (5-FU) or capecitabine. 5-FU (425 mg/ $\mathrm{m}^{2} /$ day $)$ and leucovorin $\left(20 \mathrm{mg} / \mathrm{m}^{2} /\right.$ day $)$ were administered intravenously for 5 days during the first and fifth weeks of radiotherapy. Oral capecitabine $\left(825 \mathrm{mg} / \mathrm{m}^{2} /\right.$ day $)$ was administered twice daily during the period of radiotherapy. All patients underwent curative resection with 8 weeks of intervals from the completion of nCRT. Surgery was performed by experienced colorectal surgeons following principles of total mesorectal excision.

\section{MRI Staging and Pathological Staging}

All MRI reports were retrospectively reviewed. Tumors with definite invasion to mesorectal fascia were defined as T4 stage. Tumors with invasion into perirectal fat tissues without reaching the mesorectal fascia were defined as T3 stage. Tumors without evidence of invasion to perirectal fat tissue and confined in muscle layer or within the mucosa were defined as T1-T2. Tumors with one or more probable or definite metastatic lymph node enlargement were defined as $\mathrm{N}+$. Pathological CR was defined as ypTxN0M0.

\section{${ }^{18}$ F-FDG PET/CT Imaging and Interpretation}

Baseline 18F-FDG PET/CT was performed at 7-10 days before the induction of nCRT. Follow-up PET/CT was performed at 45 weeks after the completion of nCRT. Patients fasted for at least $6 \mathrm{~h}$ before the PET/CT study. Blood glucose levels were measured. They were required to be $<200 \mathrm{mg} / \mathrm{dl}$. Whole-body PET and unenhanced CT images were acquired using a PET/CT scanner (Discovery STE, GE Healthcare, Milwaukee, WI, USA). Whole-body CT was performed using a 16-slice helical CT with 30-170 mAs adjusted to the patient's body weight at $140 \mathrm{kVp}$ 
and $3.75-\mathrm{mm}$ section width. After the CT scan, an emission scan was performed from the thigh to the head for $2.5 \mathrm{~min}$ per frame in three-dimensional mode, at $60 \mathrm{~min}$ after the intravenous injection of $18 \mathrm{~F}-\mathrm{FDG}(5.5 \mathrm{MBq} / \mathrm{kg})$. PET images were reconstructed using $\mathrm{CT}$ for attenuation correction using ordered subsets expectation-maximization algorithm (20 subsets, two iterations) with a voxel size of $3.9 \times 3.9 \times 3.3 \mathrm{~mm}$. The SUV was normalized to the patient's body weight. Volumebased assessments of 18F-FDG PET/CT were performed using a volume viewer software on a GE Advantage Workstation version 4.4. We placed a volume of interest over the primary tumor using a threshold SUV of 2.5 for tumor segmentation because this cutoff value is generally considered to be indicative of malignant tissue regardless of the scanner (15). The software then measured SUVmax, mean SUV ( $\left.\mathrm{SUV}_{\text {mean }}\right)$, a standard deviation of SUV $\left(S_{S V}\right)$, and MTV. TLG was calculated by multiplying SUV $\mathrm{SU}_{\text {mean }}$ by MTV. $\Delta$ value was defined as the difference between pre-PET/ CT parameters and post-PET/CT parameters divided by pre-PET/CT parameters.

\section{Statistical Analysis}

Statistical differences between groups were calculated using Student's t-tests for continuous variables and $\chi^{2}$ test or Fisher's exact test for categorical variables. Patients were divided to training and validation groups by random sampling with a ratio of 2:1. Univariable logistic regression analysis for the training group was performed with cell differentiation, and pre- and postvalue of CEA, MRI T stage, MRI N stage, $\mathrm{SUV}_{\text {max }}, \mathrm{SUV}_{\text {mean }}, \mathrm{SUV}_{\text {sd }}, \mathrm{MTV}$, and TLG. Multivariable regression analysis for the training group was performed using variables showing significant associations $(p<$ 0.05 ) with pCR in univariable regression analysis.

Patients were randomly assigned to a training group or a validation group with a ratio of $2: 1$. Predictive models were constructed using a training group and evaluated the efficacy in a validation group. A nomogram was established based on results of multivariable regression analysis. Other models that excluded PET/CT parameters in explanatory variables were also fitted and compared with the nomogram. The model containing CEA only, CEA with MRI staging, and CEA with MRI staging and PET/CT parameters as explanatory variables were named as "CEA" model, "CEA + MRI" model, and "CEA + MRI + PET/CT" model, respectively. Performances of these models were evaluated in terms of sensitivity, specificity, accuracy, and area under the receiver operating characteristic curve (AUC). Survival analyses were performed using the Kaplan-Meier method. Survival differences between groups were evaluated using the log-rank test. All statistical analyses were performed using $\mathrm{R}$ version 3.5.0. software (http://www.r-project.org, R Foundation for Statistical Computing, Vienna, Austria). All $p<0.05$ were considered statistically significant.

\section{RESULTS}

Among the 318 patients with rectal adenocarcinoma who underwent curative resection, 145 (45.6\%) performed sequential
${ }^{18}$ F-FDG PET/CT before and after nCRT. After excluding 3 (2.1\%) patients who underwent emergent surgery due to obstruction, 3 $(2.1 \%)$ patients who had multiple primary colorectal cancers, and $2(1.4 \%)$ patients who were diagnosed as metastatic diseases at the post-PET/CT, a total of 137 patients were finally recruited (Table 1). The number of patients who achieved pCR was 17 (12.4\%). The median pre-CEA was $1.5 \mathrm{ng} / \mathrm{ml}$ in the $\mathrm{pCR}$ group and $2.9 \mathrm{ng} / \mathrm{ml}$ in the non-pCR group $(p=0.005)$. The pCR group also had significantly lower post-CEA (1.0 vs. $1.6 \mathrm{ng} / \mathrm{ml}, p=$ $0.012)$. The number of patients with post-MRI Tx was $8(47.1 \%)$ in the pCR group and $17(14.2 \%)$ in the non-pCR group $(p=0.001)$. Proportions of patients with post-MRI N(-) stage were not significantly different between the two groups $(17.6 \%$ vs. $5.0 \%$, $p=0.148)$. Moreover, pre-PET/CT parameters showed no significant differences between the two groups. However, post$\mathrm{SUV}_{\text {max }}, \mathrm{SUV}_{\text {mean }}, \mathrm{SUV}_{\mathrm{sd}}, \mathrm{MTV}$, and TLG were significantly lower in the pCR group than in the non-pCR group.

In comparison between training group containing 91 (66.4\%) patients and validation group containing 46 (33.6\%) patients, age, sex, body mass index, pre- and post-CEA, cell differentiation, pre- and post- MRI T and $\mathrm{N}$ staging, and preand post- PET/CT parameters showed no significant. The rate of pCR was $11.0 \%(10 / 91)$ in the training group and $15.2 \%(7 / 46)$ in the validation group. Univariable regression analysis of the training group revealed that pre-CEA, post-CEA, post-MRI $T$ staging, post-SUVmax, post-SUVmean, post-MTV, post-TLG, and $\Delta S U V_{\max }$ were significantly correlated with $\mathrm{pCR}$. In multivariable regression analysis using these variables, postCEA, post-MRI T staging, post-SUV $\mathrm{Smax}_{\text {, }}$ and post-MTV were independent predictors for pCR (Table 2). A nomogram incorporating these independent predictors was developed (Figure 1). Each value or category within these factors was assigned a score on the point scale bar. After obtaining the total score, a vertical line was drawn downwards from the total point scale bar to produce probability for pCR. For example, suppose a virtual patient whose post-CEA is $1 \mathrm{ng} / \mathrm{ml}$, post-MRI T stage is Tx, post-SUV $\max$ is 4 , and post-MTV is 20 (Figure 2). The points for each item are $86,50,74$, and 78, respectively, and the total point is 288 . Finally, the probability for pCR corresponding to the total point of 288 is 0.82 .

Sensitivity, specificity, accuracy, and AUC of nomogram were $0.882,0.808$, and 0.884 , respectively (Table 3 and Figure 3A). To validate the nomogram, it was adopted to patients in the validation group to evaluate the performance (Table 4 and Figure 3B). Sensitivity, specificity, accuracy, and AUC of the nomogram were $0.857,0.781,0.783$, and 0.828 , respectively.

Without PET/CT parameters, we also construed other prediction models including "CEA" model and "CEA + MRI" model using the training group. "CEA" model contained postCEA only, and "CEA + MRI" model had post-CEA with postMRI T staging as explanatory variables. AUC was 0.689 for the "CEA" model and 0.831 for the "CEA + MRI" model, lower than that of the nomogram at 0.884 (Table 3 and Figure $3 \mathbf{A}$ ). With the validation group, the AUC was 0.544 for the "CEA" model and 0.777 for the "CEA + MRI" model, also lower than that of the nomogram at 0.828 (Table 4 and Figure 3B). 
TABLE 1 | Comparisons of clinicopathological characteristics of patients who achieved pathological complete response and those who did not.

\begin{tabular}{|c|c|c|c|}
\hline & pCR $(n=17)$ & Non-pCR $(n=120)$ & $p$-value \\
\hline Age, $\mathrm{n}(\%)$ & & & 0.368 \\
\hline$\geq 65$ & $2(11.8)$ & $30(25.0)$ & \\
\hline$<65$ & $15(88.2)$ & $90(75.0)$ & \\
\hline Sex, n (\%) & & & 0.982 \\
\hline Male & $11(64.7)$ & $82(68.3)$ & \\
\hline Female & $6(35.3)$ & $38(31.7)$ & \\
\hline BMl, mean (SD), kg/m² & $25.1(3.4)$ & $24.2(3.0)$ & \\
\hline Pre-CEA, median (IQR), ng/ml & $1.5(1.1-2.6)$ & $2.9(1.7-4.1)$ & 0.005 \\
\hline Post-CEA, median (IQR), ng/m & $1.0(0.6-1.5)$ & $1.6(1.0-2.3)$ & 0.012 \\
\hline Cell differentiation, n (\%) & & & 1.000 \\
\hline WD/MD & $15(88.2)$ & $104(86.7)$ & \\
\hline PD/Mucinous & $2(11.8)$ & $16(13.3)$ & \\
\hline Pre-MRI T stage, n (\%) & & & 0.129 \\
\hline $\mathrm{T} 1$ or $\mathrm{T} 2$ & $6(35.3)$ & $19(15.8)$ & \\
\hline сT3 & $8(47.1)$ & $64(53.3)$ & \\
\hline cT4 & $3(17.6)$ & $37(30.8)$ & \\
\hline Pre-MRI N stage, n (\%) & & & 0.821 \\
\hline $\mathrm{N}-$ & $1(5.9)$ & $2(1.7)$ & \\
\hline $\mathrm{N}+$ & $16(94.1)$ & $118(98.3)$ & \\
\hline Post-MRI T stage, n (\%) & & & 0.001 \\
\hline Tx & $8(47.1)$ & $17(14.2)$ & \\
\hline $\mathrm{T} 1$ or $\mathrm{T} 2$ & $6(35.3)$ & $21(17.5)$ & \\
\hline T3 & $2(11.8)$ & $65(54.2)$ & \\
\hline T4 & $1(5.9)$ & $17(14.2)$ & \\
\hline Post-MRI N stage, n (\%) & & & 0.148 \\
\hline $\mathrm{N}-$ & $3(17.6)$ & $6(5.0)$ & \\
\hline $\mathrm{N}+$ & $14(82.4)$ & $114(95.0)$ & \\
\hline Pathologic T stage, n (\%) & & & $<0.001$ \\
\hline ypTx & $17(100)$ & $2(1.7)$ & \\
\hline ypT1 & 0 & 4 (3.3) & \\
\hline урт2 & 0 & $50(41.7)$ & \\
\hline урT3 & 0 & $63(52.5)$ & \\
\hline ypT4 & 0 & $1(0.8)$ & \\
\hline Pathologic N stage, n (\%) & & & 0.01 \\
\hline ypNO & $17(100)$ & $76(63.3)$ & \\
\hline ypN1 & 0 & $34(28.3)$ & \\
\hline ypN2 & 0 & $10(8.3)$ & \\
\hline Pre-SUV max, median (IQR) & $13.3(10.7-16.6)$ & $14.4(9.7-17.9)$ & 0.739 \\
\hline Pre-SUV mean, $_{\text {, median (IQR) }}$ & $6.2(4.9-7.8)$ & $8.0(4.9-9.2)$ & 0.195 \\
\hline Pre-SUV ${ }_{\text {sd }}$, median (IQR) & $2.8(1.8-3.5)$ & $2.3(1.8-2.9)$ & 0.601 \\
\hline Pre-MTV, median (IQR) & $18.0(6.1-29.5)$ & $19.8(14.0-32.6)$ & 0.245 \\
\hline Pre-TLG, median (IQR) & $77.0(55.9-236.2)$ & $122.3(75.7-216.1)$ & 0.249 \\
\hline Post-SUV $V_{\max }$, median (IQR) & $3.1(2.2-4.6)$ & $6.8(4.0-9.8)$ & 0.005 \\
\hline Post-SUV ${ }_{\text {mean, }}$ median (IQR) & $2.8(2.6-3.2)$ & $3.2(2.7-3.9)$ & 0.035 \\
\hline Post-SUV ${ }_{\text {sd }}$, median (IQR) & $0.4(0.4-0.6)$ & $0.7(0.5-1.0)$ & $<0.001$ \\
\hline Post-MTV, median (IQR) & $2.4(1.3-4.8)$ & $6.1(3.8-12.7)$ & 0.020 \\
\hline Post-TLG, median (IQR) & $0.5(3.8-13.0)$ & $12.8(6.0-33.1)$ & 0.019 \\
\hline$\Delta S \mathrm{SV}_{\max }$, median $(\mathrm{IQR})$ & $72.1(57.5-76.5)$ & $60.0(48.2-69.8)$ & 0.015 \\
\hline$\Delta S U V_{\text {mean }}$, median $(\mathrm{IQR})$ & $60.6(43.9-72.2)$ & 44.7 (34.1-58.3) & 0.022 \\
\hline$\Delta \mathrm{SUV}_{\mathrm{sd}}$, median $(\mathrm{IQR})$ & $81.2(66.3-87.5)$ & $66.7(54.9-78.5)$ & 0.005 \\
\hline$\Delta \mathrm{MTV}$, median (IQR) & $82.9(50.3-92.1)$ & 79.5 (57.9-90.8) & 0.659 \\
\hline$\Delta T L G$, median (IQR) & 93.8 (79.1-96.8) & 87.7 (75.7-95.2) & 0.221 \\
\hline
\end{tabular}

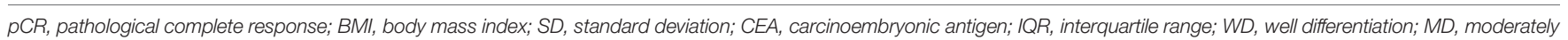
differentiation; PD, poorly differentiation; MRI, magnetic resonance imaging; SUV, standardized uptake value; MTV, metabolic tumor volume; TLG, total lesion glycolysis.

The median follow-up period was 87 months. Oncological outcomes were compared between pCR and non-pCR groups (Figure 4). Three-year disease-free survival rate was 100\% for the pCR group and $76.3 \%$ for the non-pCR group $(p=0.02)$. Threeyear overall survival was $100 \%$ for the pCR group and $93.2 \%$ for the non-pCR group $(p=0.23)$.

\section{DISCUSSION}

The clinical evidence for excellent prognosis of patients with $\mathrm{pCR}$ has been well established (17). Our data also revealed that 5-year disease-free survival and overall survival of patients with $\mathrm{pCR}$ were $100 \%$. Therefore, we could infer that oncological outcomes 
TABLE 2 | Multivariable regression models for pathologic complete response in the training group.

\begin{tabular}{lccc}
\hline & Odds ratio & 95\% confidence interval & $\boldsymbol{p}$-value \\
\hline $\begin{array}{l}\text { Post-CEA } \\
\text { Post-MRI T stage }\end{array}$ & 2.503 & $1.107-6.918$ & 0.048 \\
T1-2 vs. Tx & 0.960 & $0.240-3.823$ & 0.954 \\
T3 vs. Tx & 5.312 & $1.878-64.93$ & 0.011 \\
T4 vs. Tx & 8.893 & $0.730-110.0$ & 0.152 \\
Post-SUV & 1.547 & $1.068-2.493$ & 0.041 \\
Post-MTV & 1.187 & $1.113-1.486$ & 0.039 \\
\hline
\end{tabular}

CEA, carcinoembryonic antigen; MRI, magnetic resonance imaging; SUV, standardized uptake value; MTV, metabolic tumor volume.

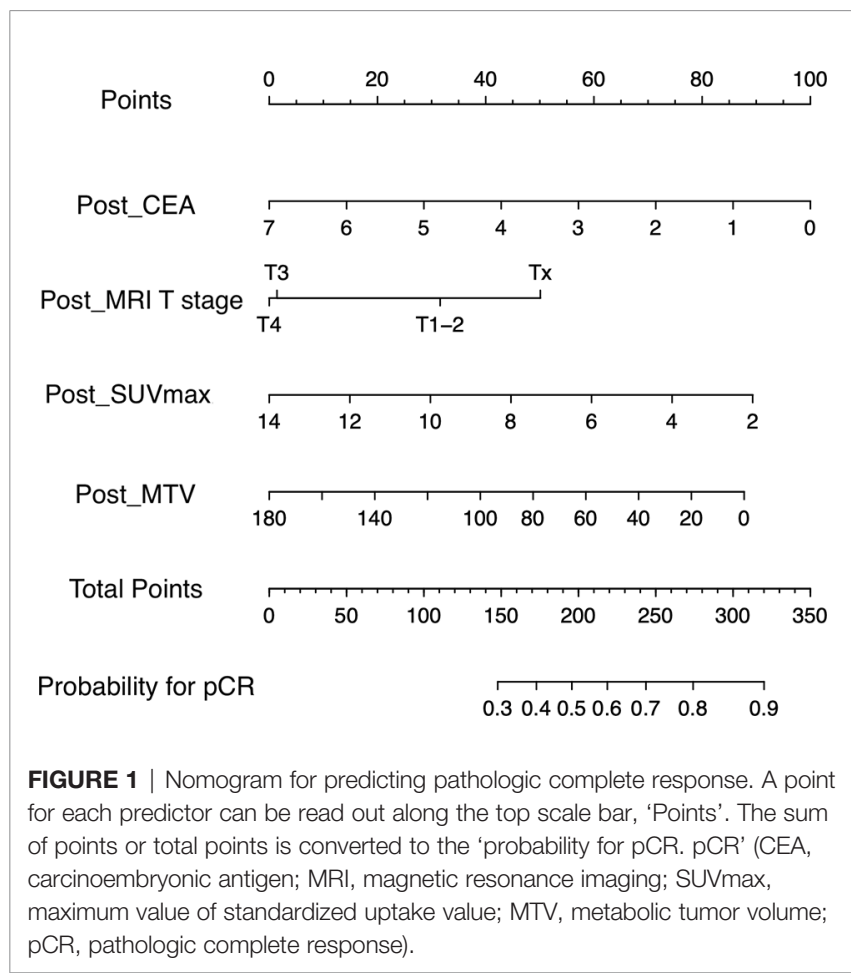

of these patients may not be compromised by the application of organ-sparing strategies. To select these patients accurately, we considered several clinical variables. Most of all, this study evaluated predictive values of semiquantitative volumetric and metabolic parameters derived from pre- and post-PET/CT for pCR in patients with LARC who underwent nCRT. Our findings demonstrated that not pre-PET/CT, but post-PET/CT parameters were significantly correlated with $\mathrm{pCR}$. Our results also revealed that post-SUV max and post-MTV and CEA and post-MRI $\mathrm{T}$ staging were independent predictors in multivariable regression analysis. A nomogram incorporating post-PET/CT parameters with post-CEA and post-MRI T staging features was successfully developed and validated, with predictive performances of AUC 0.884 and 0.828 for the training group and the validation group, respectively. Because performances of the nomogram were better than other models that did not contain PET/CT parameters, the addition of PET/

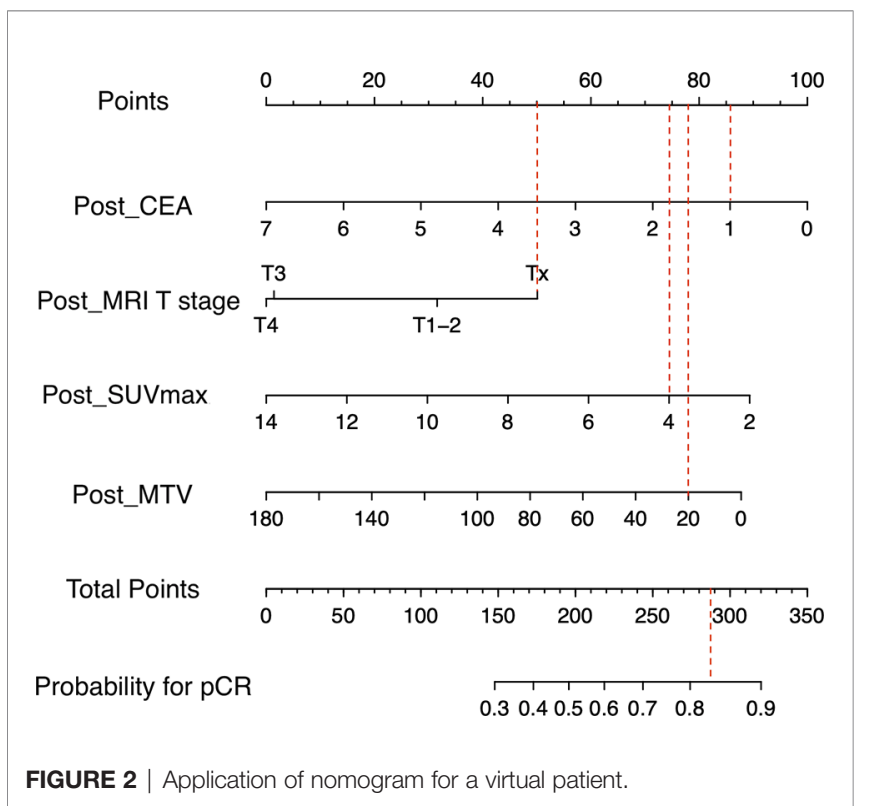

CT variables, especially post-SUV $\mathrm{max}_{\max }$ and MTV, could improve a model's predictive power for pCR.

Interestingly, our results demonstrated that pre-nCRT variables were not correlated with $\mathrm{pCR}$ in multivariable regression. Ryan et al. have performed a systematic review for predicting pCR using pre-nCRT variables in LARC (18). They selected 85 articles addressing the prediction of PCR with clinical, radiological, and molecular characteristics. Although some studies in their review suggested that pre-CEA, pre-MRI parameter, specific mutation profiles, and/or protein expression profiles of tumors were associated with pCR, no robust solitary pre-nCRT marker was identified. Moreover, no studies have confirmed the significant predictability of pre-PET/CT parameters for $\mathrm{pCR}$ in the review, corresponding to results of the present study.

Because radiation-related tumor shrinkage effect is timedependent phenomenon, the optimal timing of restaging and surgery after nCRT completion has long been a critical issue. Although a minimum of 6-8 weeks interval to surgery is commonly recommended to maximize a tumor downsizing and $\mathrm{pCR}$ rates, a consensus or clinical guidelines regarding the best timing for assessment of tumor response to nCRT is still lacking. Perez et al. conducted a clinical trial to estimate the metabolic activity at 6 and 12 weeks after nCRT by PET/CT (19). The patients were treated with long-course nCRT and underwent three PET/CT at baseline, 6 weeks, and 12 weeks from nCRT completion. In the results of the study, SUV $\max$ decreased until 6 weeks for both good responders and bad responders, remained identical or further decreased from 6 to 12-week PET/CT imaging for good responders, and showed a rise from 6 to 12week PET/CT imaging for bad responders. This study also showed that a decrease between early $(1 \mathrm{~h})$ and late $(3 \mathrm{~h})$ $\mathrm{SUV}_{\max }$ at 6-week PET/CT imaging could predict good responders with an accuracy of $67 \%$. Gasinska et al. also 
TABLE 3 | Performances of models for the training group.

\begin{tabular}{|c|c|c|c|c|}
\hline & Sensitivity & Specificity & Accuracy & AUC \\
\hline CEA & 0.706 & 0.467 & 0.518 & 0.689 \\
\hline $\mathrm{CEA}+\mathrm{MRI}$ & 0.882 & 0.858 & 0.839 & 0.831 \\
\hline CEA + MRI + PET/CT (nomogram) & 0.882 & 0.808 & 0.848 & 0.884 \\
\hline
\end{tabular}

CEA, carcinoembryonic antigen; MRI, magnetic resonance imaging; PET/CT, positron emission tomography/computed tomography.

A

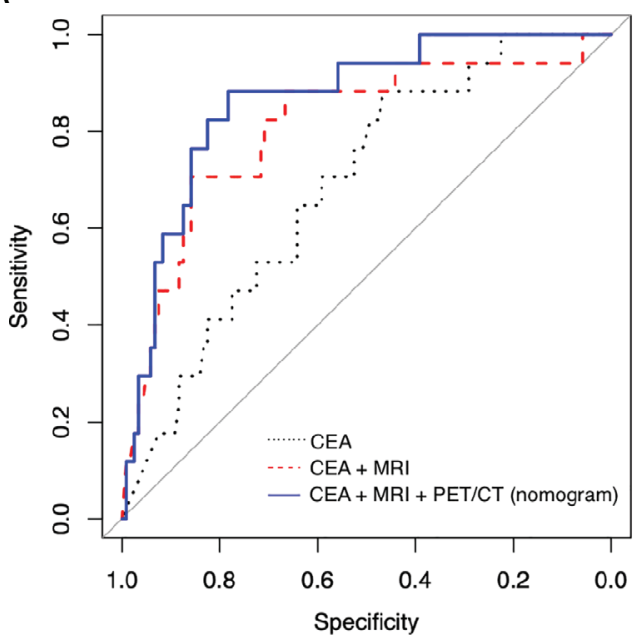

B

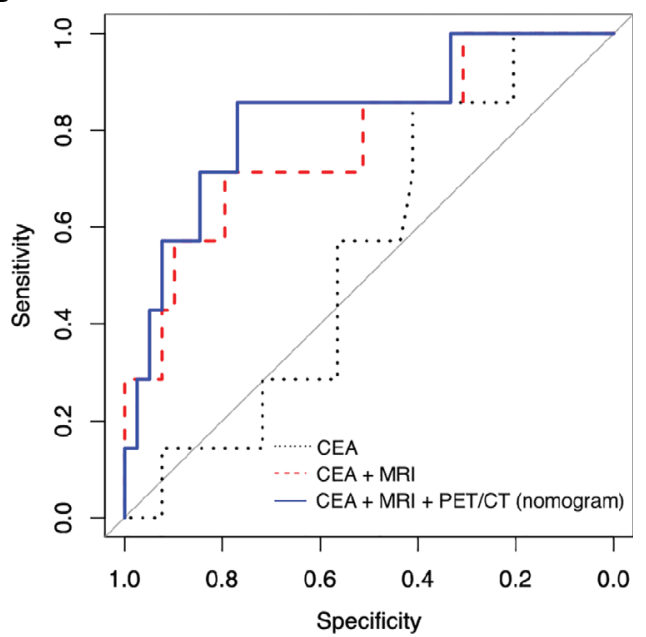

FIGURE 3 | Receiver operating characteristic (ROC) curve analysis to evaluate the predictive power of models in (A) training group and (B) validation group. (CEA, carcinoembryonic antigen; MRI, magnetic resonance imaging; PET/CT, positron emission tomography/computed tomography).

showed that repopulation of tumor cells occurred 4 weeks after nCRT completion (20). In this study, post-PET/CT was conducted 4-5 weeks after nCRT completion based on the results of the previously said studies. However, to establish robust evidence for an optimal timing for reassessment by PET/CT after long-course nCRT completion, a well-designed randomized controlled trials should be conducted.

Although follow-up or restaging imaging with MRI has been routinely recommended in clinical guidelines, the clinical benefit and usefulness of restaging PET/CT have yet to be established $(12,21,22)$. Recently, some studies have shown that the predictive power of post-nCRT variables may be better than those of pre-nCRT variables, meaning that post-nCRT clinical or imaging features could provide more valuable information regarding the response to nCRT (23-27). Moreover, restaging with PET/CT could even detect new metastatic lesions after longcourse nCRT in some patients with non-pCR (25).
However, as mentioned above, no modality including MRI or PET/CT was confirmed as a single significant predictor for pCR. Therefore, researchers tried to integrate several markers to improve the performance of predicting models. Ren et al. have constructed a nomogram for predicting $\mathrm{PCR}$ in patients treated by neoadjuvant mFOLFOX6 with radiotherapy, known as total neoadjuvant therapy (TNT) (28). These patients were participants in the FOWARC trial (29). Their nomogram contained variables of tumor differentiation, mesorectal fascia status evaluated by pre-MRI, regimen of nCRT, and tumor size. However, they did not consider PET/CT parameters. Although their nomogram showed good statistical performance for predicting the probability of pCR with C-index of $79.34 \%$, it might be due to a relatively high pCR rate $(17.9 \%)$ caused by more aggressive neoadjuvant therapy regimen compared to standard nCRT. Considering that high pCR rate itself could improve the accuracy of predicting models in statistics, our

TABLE 4 | Performances of models for the validation group.

\begin{tabular}{lccr}
\hline & Sensitivity & Specificity & Accuracy \\
\hline CEA & 0.857 & 0.410 & 0.501 \\
CEA + MRI & 0.714 & 0.795 & 0.783 \\
CEA + MRI + PET/CT (nomogram) & 0.857 & 0.781 & 0.544 \\
\hline
\end{tabular}

CEA, carcinoembryonic antigen; MRI, magnetic resonance imaging; PET/CT, positron emission tomography/computed tomography. 


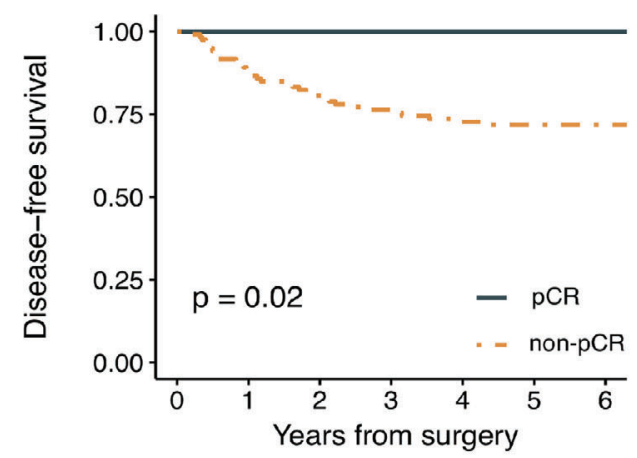

No. at risk

$\begin{array}{llllllll}\mathrm{pCR}(+) & 17 & 17 & 16 & 16 & 15 & 13 & 13\end{array}$

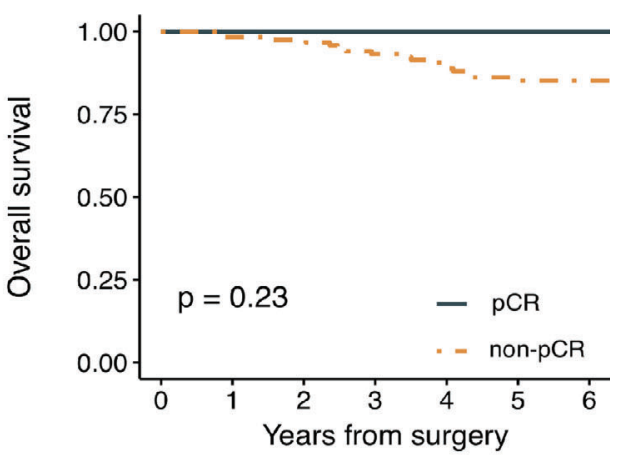

No. at risk

$\begin{array}{llllllll}\mathrm{PCR}(+) & 17 & 17 & 16 & 16 & 15 & 13 & 13\end{array}$

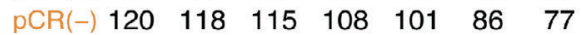

FIGURE 4 | Kaplan-Meier survival curve analysis for (A) disease-free survival and (B) overall survival in patients with or without pathologic complete response. (pCR; pathologic complete response).

nomogram showing an accuracy of $78.3 \%$ in the patient cohort with pCR rate of $12.4 \%$ might have potential to show better performance in the patient cohort treated by TNT known to induce a higher $\mathrm{pCR}$ rate.

It has been known that MRI and PET/CT have comparable diagnostic performance for the prediction of pCR (30). Joye et al. have conducted a systematic review for studies on the role of diffusion-weighted MRI and PET/CT in the prediction of $\mathrm{pCR}$ and concluded that diffusion-weighted MRI or PET/CT alone is not accurate in prediction of $\mathrm{pCR}$, although it has strength in the identification of non-pCR (31). In their study, integration of MRI and PET/CT was not considered. Because both modalities showed complimentary results in many studies, 18-F FDG PET/MRI was suggested as a solution to increase the sensitivity by adding MRI parameters to PET parameter, and the initial experience was reported recently (32). However, this technique has some disadvantages compared to other hybrid imaging techniques including lack of protocol and standardization, limited flexibility of combined PET/MRI systems, and requirement of high cost. In addition, several technical challenges such as the addition of PET components to the system in the presence of strong magnetic field from MR have remained to be widely used in clinical practices (33).

As PET/MR technically integrates PET and MRI, our nomogram statistically integrates their outputs. Because postMRI could precisely determine the tumor's depth of invasion, post-MRI T staging was a significant predictor for $\mathrm{pCR}$ in our study. However, the accuracy of post-MRI N staging was limited because MRI could only assess the size and shape of a lymph node instead of its physiologic activity. This limitation of MRI was supplemented by semiquantitative parameters of post-PET/ CT. SUV $\mathrm{max}_{\max }$ the maximum voxel value of SUV in the target lesion, is the most valuable and common parameter of PET/CT. However, it does not reproduce the whole metabolic tumor burden. In addition, it is vulnerable to various noises generated by several factors, including patient characteristics (34). MTV is a measurement of functional tumor volume with high metabolic activity. TLG is a product of MTV and mean SUV. These semiquantitative volumetric parameters could represent metabolic activity of the tumor better than $\mathrm{SUV}_{\max }(13-16)$. In recent years, several studies have analyzed predictive values of MTV and TLG for pCR in LARC (35-37). However, no parameter alone was sufficiently effective to play a secure role in selecting patients with pCR. For the above-mentioned reasons, we incorporated all parameters derived from PET/CT with MRI features into the nomogram.

This study had some limitations. First, because this study was conducted retrospectively and the cohort did not represent all consecutive patients with LARC treated in this center, the inclusion of patients might have been affected by selection bias. Second, results of this single-center analysis based on a small number of patients lacked generalizability. Especially, an external validation using a test group or patients outside this center was not performed. Third, calculating parameters of PET/CT was laborious to be easily applied to real-world practice. Moreover, as it was performed by expert nuclear medicine physicians, it may raise concerns regarding interobserver variability issues. Further welldesigned multicenter prospective studies are warranted to confirm the predictive role of this nomogram. Fourth, because the PET/CT has fundamentally limited performance on spatial resolution and the resulting partial volume effect, PET/CT parameters of the small lesions may be underestimated, and this false negativity may exaggerate the probability for pCR in a nomogram. Therefore, if the post-SUV $\mathrm{Smax}_{\text {max }}$ or post-MTV of the lesion was too low or not detected while post-MRI T stage was obviously greater than T1-T2, the results of nomogram should be cautiously interpreted.

In conclusion, post-PET/CT parameters including post$\mathrm{SUV}_{\max }$ and post-MTV have significant predictive values for pCR. A nomogram incorporating semiquantitative post-PET/CT parameters with post-MRI features could effectively select candidates for organ-sparing strategies. 


\section{DATA AVAILABILITY STATEMENT}

The raw data supporting the conclusions of this article will be made available by the authors, without undue reservation.

\section{ETHICS STATEMENT}

The studies involving human participants were reviewed and approved by Samsung Medical Center. The patients/participants provided their written informed consent to participate in this study.

\section{AUTHOR CONTRIBUTIONS}

DHP performed the statistical analysis and data interpretation and wrote a draft. JYC calculated the parameters of PET/CT.

\section{REFERENCES}

1. Sung H, Ferlay J, Siegel RL, Laversanne M, Soerjomataram I, Jemal A, et al. Global Cancer Statistics 2020: GLOBOCAN Estimates of Incidence and Mortality Worldwide for 36 Cancers in 185 Countries. CA Cancer J Clin (2021) 71(3):209-49. doi: 10.3322/caac.21660

2. Sauer R, Becker H, Hohenberger W, Rodel C, Wittekind C, Fietkau R, et al. Preoperative Versus Postoperative Chemoradiotherapy for Rectal Cancer. N Engl J Med (2004) 351(17):1731-40. doi: 10.1056/NEJMoa040694

3. Garcia-Aguilar J, Hernandez de Anda E, Sirivongs P, Lee SH, Madoff RD, Rothenberger DA. A Pathologic Complete Response to Preoperative Chemoradiation is Associated With Lower Local Recurrence and Improved Survival in Rectal Cancer Patients Treated by Mesorectal Excision. Dis Colon Rectum (2003) 46(3):298-304. doi: 10.1007/s10350-004-6545-x

4. Moore HG, Gittleman AE, Minsky BD, Wong D, Paty PB, Weiser M, et al. Rate of Pathologic Complete Response With Increased Interval Between Preoperative Combined Modality Therapy and Rectal Cancer Resection. Dis Colon Rectum (2004) 47(3):279-86. doi: 10.1007/s10350-003-0062-1

5. Habr-Gama A, Perez RO, Nadalin W, Sabbaga J, Ribeiro UJr., Silva e Sousa AHJr., et al. Operative Versus Nonoperative Treatment for Stage 0 Distal Rectal Cancer Following Chemoradiation Therapy: Long-Term Results. Ann Surg (2004) 240(4):711-7. doi: 10.1097/01.sla.0000141194.27992.32. discussion 7-8.

6. Smith JJ, Strombom P, Chow OS, Roxburgh CS, Lynn P, Eaton A, et al. Assessment of a Watch-and-Wait Strategy for Rectal Cancer in Patients With a Complete Response After Neoadjuvant Therapy. JAMA Oncol (2019) 5(4): e185896-e. doi: 10.1001/jamaoncol.2018.5896

7. Dizdarevic E, Frøstrup Hansen T, Pløen J, Henrik Jensen L, Lindebjerg J, Rafaelsen S, et al. Long-Term Patient-Reported Outcomes After High-Dose Chemoradiation Therapy for Nonsurgical Management of Distal Rectal Cancer. Int J Radiat Oncol Biol Phys (2020) 106(3):556-63. doi: 10.1016/ j.ijrobp.2019.10.046

8. Kundel Y, Brenner R, Purim O, Peled N, Idelevich E, Fenig E, et al. Is Local Excision After Complete Pathological Response to Neoadjuvant Chemoradiation for Rectal Cancer an Acceptable Treatment Option? Dis Colon Rectum (2010) 53(12):1624-31. doi: 10.1007/DCR.0b013e3181f5b64d

9. Althumairi AA, Gearhart SL. Local Excision for Early Rectal Cancer: Transanal Endoscopic Microsurgery and Beyond. J Gastrointest Oncol (2015) 6(3):296. doi: 10.3978/j.issn.2078-6891.2015.022

10. Bökkerink GM, de Graaf EJ, Punt CJ, Nagtegaal ID, Rütten H, Nuyttens JJ, et al. The CARTS Study: Chemoradiation Therapy for Rectal Cancer in the Distal Rectum Followed by Organ-Sparing Transanal Endoscopic Microsurgery. BMC Surg (2011) 11(1):1-7. doi: 10.1002/bjs.9809

11. Litvak A, Cercek A, Segal N, Reidy-Lagunes D, Stadler ZK, Yaeger RD, et al. False-Positive Elevations of Carcinoembryonic Antigen in Patients With a
YBC designed the core conception and guided the whole process. YBC, WYL, SHY, HCK, JWH, YAP, and JKS constructed and collected the clinical database. YBC did a critical revision for intellectual content. All authors contributed to the article and approved the submitted version.

\section{FUNDING}

This research was supported by a grant of the Korea Health Technology R\&D project through the Korea Health Industry Development Institute (KHIDI), funded by the Ministry of Health \& Welfare, Republic of Korea (grant number: HR20C0025). This work was supported by the BK21 FOUR Project.
History of Resected Colorectal Cancer. J Natl Compr Canc Netw (2014) 12 (6):907-13. doi: 10.6004/jnccn.2014.0085

12. Beets-Tan RGH, Lambregts DMJ, Maas M, Bipat S, Barbaro B, Curvo-Semedo L, et al. Magnetic Resonance Imaging for Clinical Management of Rectal Cancer: Updated Recommendations From the 2016 European Society of Gastrointestinal and Abdominal Radiology (ESGAR) Consensus Meeting. Eur Radiol (2018) 28(4):1465-75. doi: 10.1007/s00330-017-5026-2

13. Albano D, Bosio G, Pagani C, Re A, Tucci A, Giubbini R, et al. Prognostic Role of Baseline 18F-FDG PET/CT Metabolic Parameters in Burkitt Lymphoma. Eur J Nucl Med Mol Imaging (2019) 46(1):87-96. doi: 10.1007/s00259-0184173-2

14. Liao S, Penney BC, Wroblewski K, Zhang H, Simon CA, Kampalath R, et al. Prognostic Value of Metabolic Tumor Burden on 18 F-FDG PET in Nonsurgical Patients With non-Small Cell Lung Cancer. Eur J Nucl Med Mol Imaging (2012) 39(1):27-38. doi: 10.1007/s00259-011-1934-6

15. Lee JW, Kang CM, Choi HJ, Lee WJ, Song SY, Lee J-H, et al. Prognostic Value of Metabolic Tumor Volume and Total Lesion Glycolysis on Preoperative 18F-FDG PET/CT in Patients With Pancreatic Cancer. J Nucl Med (2014) 55 (6):898-904. doi: 10.2967/jnumed.113.131847

16. Liao S, Penney BC, Zhang H, Suzuki K, Pu Y. Prognostic Value of the Quantitative Metabolic Volumetric Measurement on 18F-FDG PET/CT in Stage IV Nonsurgical Small-Cell Lung Cancer. Acad Radiol (2012) 19(1):6977. doi: 10.1016/j.acra.2011.08.020

17. Sell NM, Qwaider YZ, Goldstone RN, Cauley CE, Cusack JC, Ricciardi R, et al. Ten-Year Survival After Pathologic Complete Response in Rectal Adenocarcinoma. J Surg Oncol (2021) 123(1):293-8. doi: 10.1002/jso.26247

18. Ryan JE, Warrier SK, Lynch AC, Ramsay RG, Phillips WA, Heriot AG. Predicting Pathological Complete Response to Neoadjuvant Chemoradiotherapy in Locally Advanced Rectal Cancer: A Systematic Review. Colorectal Dis (2016) 18(3):234-46. doi: 10.1111/codi.13207

19. Perez RO, Habr-Gama A, São Julião GP, Gama-Rodrigues J, Sousa AHJr., Campos FG, et al. Optimal Timing for Assessment of Tumor Response to Neoadjuvant Chemoradiation in Patients With Rectal Cancer: Do All Patients Benefit From Waiting Longer Than 6 Weeks? Int J Radiat Oncol Biol Phys (2012) 84(5):1159-65. doi: 10.1016/j.ijrobp.2012.01.096

20. Gasinska A, Richter P, Darasz Z, Niemiec J, Bucki K, Malecki K, et al. GenderRelated Differences in Repopulation and Early Tumor Response to Preoperative Radiotherapy in Rectal Cancer Patients. J Gastrointest Surg (2011) 15(9):1568-76. doi: 10.1007/s11605-011-1589-4

21. You YN, Hardiman KM, Bafford A, Poylin V, Francone TD, Davis K, et al. The American Society of Colon and Rectal Surgeons Clinical Practice Guidelines for the Management of Rectal Cancer. Dis Colon Rectum (2020) 63(9):1191-222. doi: 10.1097/dcr.0000000000001762

22. Glynne-Jones R, Wyrwicz L, Tiret E, Brown G, Rödel C, Cervantes A, et al. Rectal Cancer: ESMO Clinical Practice Guidelines for Diagnosis, Treatment 
and Follow-Up. Ann Oncol (2017) 28(suppl_4):iv22-40. doi: 10.1093/annonc/ $\mathrm{mdx} 224$

23. Sun $\mathrm{Y}$, Chi P, Lin H, Lu X, Huang Y, Xu Z, et al. A Nomogram Predicting Pathological Complete Response to Neoadjuvant Chemoradiotherapy for Locally Advanced Rectal Cancer: Implications for Organ Preservation Strategies. Oncotarget (2017) 8(40):67732-43. doi: 10.18632/oncotarget.18821

24. Sorenson E, Lambreton F, Yu JQ, Li T, Denlinger CS, Meyer JE, et al. Impact of PET/CT for Restaging Patients With Locally Advanced Rectal Cancer After Neoadjuvant Chemoradiation. J Surg Res (2019) 243:242-8. doi: 10.1016/ j.jss.2019.04.080

25. Hendrick LE, Buckner JD, Guerrero WM, Shibata D, Hinkle NM, Monroe JJ, et al. What Is the Utility of Restaging Imaging for Patients With Clinical Stage I//III Rectal Cancer After Completion of Neoadjuvant Chemoradiation and Prior to Proctectomy? Am Surg (2021) 87(2):242-7. doi: 10.1177/0003134820950298

26. Kim SJ, Chang S. Volumetric Parameters Changes of Sequential 18F-FDG PET/CT for Early Prediction of Recurrence and Death in Patients With Locally Advanced Rectal Cancer Treated With Preoperative Chemoradiotherapy. Clin Nucl Med (2015) 40(12):930-5. doi: 10.1097/ rlu.0000000000000917

27. Altini C, Niccoli Asabella A, De Luca R, Fanelli M, Caliandro C, Quartuccio N, et al. Comparison of (18)F-FDG PET/CT Methods of Analysis for Predicting Response to Neoadjuvant Chemoradiation Therapy in Patients With Locally Advanced Low Rectal Cancer. Abdom Imaging (2015) 40(5):1190-202. doi: 10.1007/s00261-014-0277-8

28. Ren DL, Li J, Yu HC, Peng SY, Lin WD, Wang XL, et al. Nomograms for Predicting Pathological Response to Neoadjuvant Treatments in Patients With Rectal Cancer. World J Gastroenterol (2019) 25(1):118-37. doi: 10.3748/wjg.v25.i1.118

29. Deng Y, Chi P, Lan P, Wang L, Chen W, Cui L, et al. Neoadjuvant Modified FOLFOX6 With or Without Radiation Versus Fluorouracil Plus Radiation for Locally Advanced Rectal Cancer: Final Results of the Chinese FOWARC Trial. J Clin Oncol (2019) 37(34):3223-33. doi: 10.1200/jco.18.02309

30. Lee SW, Jeong SY, Kim K, Kim SJ. Direct Comparison of F-18 FDG PET/CT and MRI to Predict Pathologic Response to Neoadjuvant Treatment in Locally Advanced Rectal Cancer: A Meta-Analysis. Ann Nucl Med (2021) 35(9):103847. doi: 10.1007/s12149-021-01639-y

31. Joye I, Deroose CM, Vandecaveye V, Haustermans K. The Role of DiffusionWeighted MRI and 18F-FDG PET/CT in the Prediction of Pathologic Complete Response After Radiochemotherapy for Rectal Cancer: A Systematic Review. Radiother Oncol (2014) 113(2):158-65. doi: 10.1016/ j.radonc.2014.11.026
32. Cho N, Im SA, Cheon GJ, Park IA, Lee KH, Kim TY, et al. Integrated (18)FFDG PET/MRI in Breast Cancer: Early Prediction of Response to Neoadjuvant Chemotherapy. Eur J Nucl Med Mol Imaging (2018) 45 (3):328-39. doi: 10.1007/s00259-017-3849-3

33. Muzic RFJr., DiFilippo FP. Positron Emission Tomography-Magnetic Resonance Imaging: Technical Review. Semin Roentgenol (2014) 49(3):24254. doi: 10.1053/j.ro.2014.10.001

34. Im HJ, Bradshaw T, Solaiyappan M, Cho SY. Current Methods to Define Metabolic Tumor Volume in Positron Emission Tomography: Which One is Better? Nucl Med Mol Imaging (2018) 52(1):5-15. doi: 10.1007/s13139-0170493-6

35. Fernando S, Lin M, Pham TT, Chong S, Ip E, Wong K, et al. Prognostic Utility of Serial (18)F-FDG-PET/CT in Patients With Locally Advanced Rectal Cancer Who Underwent Tri-Modality Treatment. Br J Radiol (2020) 93 (1105):20190455. doi: 10.1259/bjr.20190455

36. Okuno T, Kawai K, Koyama K, Takahashi M, Ishihara S, Momose T, et al. Value of FDG-PET/CT Volumetry After Chemoradiotherapy in Rectal Cancer. Dis Colon Rectum (2018) 61(3):320-7. doi: 10.1097/dcr.0000000000000959

37. Dos Anjos DA, Perez RO, Habr-Gama A, São Julião GP, Vailati BB, Fernandez LM, et al. Semiquantitative Volumetry by Sequential PET/CT May Improve Prediction of Complete Response to Neoadjuvant Chemoradiation in Patients With Distal Rectal Cancer. Dis Colon Rectum (2016) 59(9):805-12. doi: 10.1097/dcr.0000000000000655

Conflict of Interest: The authors declare that the research was conducted in the absence of any commercial or financial relationships that could be construed as a potential conflict of interest.

Publisher's Note: All claims expressed in this article are solely those of the authors and do not necessarily represent those of their affiliated organizations, or those of the publisher, the editors and the reviewers. Any product that may be evaluated in this article, or claim that may be made by its manufacturer, is not guaranteed or endorsed by the publisher.

Copyright (๑ 2021 Pyo, Choi, Lee, Yun, Kim, Huh, Park, Shin and Cho. This is an open-access article distributed under the terms of the Creative Commons Attribution License (CC BY). The use, distribution or reproduction in other forums is permitted, provided the original author(s) and the copyright owner(s) are credited and that the original publication in this journal is cited, in accordance with accepted academic practice. No use, distribution or reproduction is permitted which does not comply with these terms. 